\title{
Los hongos como elementos clave en la productividad del suelo, la agricultura y el bienestar social
}

\author{
Fungi as key elements in soil productivity, agriculture, \\ and social wellness
}

\author{
Krystal Zúñiga-Castro \\ Gerald Quirós-Cedeño
}

DOl: https://doi.org/10.22458/rb.v32i1.3548

Recibido- Received: 02/02/2021 / Corregido- Revised: 20/02/2021 / Aceptado- Accepted: 25/03/2021

\section{RESUMEN}

El desconocimiento y manejo incorrecto de la tierra en ciertas prácticas agrícolas representa un problema para organismos que contribuyen a la salud del suelo, como los hongos. El objetivo de la investigación es demostrar la importancia del uso de los hongos como elementos clave en la productividad del suelo, la agricultura y el bienestar social e incentivar su uso, conservación y protección. El estudio se llevó a cabo en un sendero de 1,5km en Finca Boquete-Sibü, Pérez Zeledón, un Bosque Pluvial Montano Bajo, donde se observaron diferentes especies de cuerpos fructíferos por nueve meses durante la época seca y lluviosa, anotando su nombre, sustrato, altitud, y se investigaron por medio de revisión bibliográfica sus funciones para la agricultura, el suelo y el bien social. Se observaron 25 individuos de 17 especies de hongos, los cuales tienen como funciones ser los principales descomponedores de la materia orgánica y recicladores de nutrientes, crear micorrizas, ser fijadores de nitrógeno y controladores de plagas, además de utilizarse para la biorremediación y como indicadores de la calidad del suelo. Adicionalmente, pueden ser empleados como alimento, medicina, en el arte y en el turismo. El uso responsable de recursos naturales como los hongos permitirá la conservación del suelo y del ecosistema en general, además de aumentar la seguridad alimentaria y el bienestar social.

Palabras clave: hongos; suelo; micorrizas; descomposición; biorremediación; bienestar social.

\section{ABSTRACT}

The lack of knowledge and the incorrect land management in certain agricultural practices represent a problem for organisms that contribute to soil health, such as fungi. The objective of this research is to demonstrate the importance of the use of fungi as key elements in soil productivity, agriculture, and social wellness to encourage their use, conservation, and protection. The study was carried out in a 1,5km transect at Finca Boquete-Sibü, Pérez Zeledón, a Low Montane Rain Forest, where different species of fruiting bodies were observed for nine months during the dry and rainy season. During this period, information such as names, substratum, and altitude was recorded, and, through a review of literature, their functions for agriculture, soil and social wellness were examined.We observed 25 individuals of 17 species of mushrooms, which are the main decomposers of organic matter and recyclers of nutrients. They create mycorrhizae, are nitrogen fixers and pest controllers, and are also used for bioremediation and as indicators of soil quality. Moreover, they can be used as food, medicine, art, or tourism. The responsible use of natural resources such as fungi will allow the conservation of the soil and the ecosystem in general, in addition to increasing food security and social wellness.

Key words: mushrooms; soil; mycorrhizae; decomposition; bioremediation; social wellness.

* Investigadora Independiente. Estudiante de Manejo de Recursos Naturales, Universidad Estatal a Distancia, Costa Rica. krystal.zuniga@uned.cr ID: https://orcid.org/0000-0003-4032-2330

**Investigador Independiente. Estudiante de Manejo de Recursos Naturales, Universidad Estatal a Distancia, Costa Rica.gerald.quiros@uned.cr ID: https://orcid.org/0000-0002-0858-7016 


\section{Introducción}

El suelo sufre graves daños por los sistemas de producción intensivos ante la falta de conocimiento de las características del recurso edáfico, así como por no generarse un buen cuidado del mismo; la tercera parte de la superficie terrestre se dedica a la agricultura, la cual ejerce una degradación y mayor presión en los suelos, así como contaminación, erosión, pérdida de las características del recurso y de aspectos culturales relacionados (Burbano, 2016).

Existen millones de formas de vida en una pequeña porción del suelo que permiten el funcionamiento de los ecosistemas, los diferentes ciclos biogeoquímicos y el bienestar social. Entre ellas se encuentran los hongos, organismos descomponedores, principalmente de la lignina y la celulosa (componentes primarios de la madera), así como recicladores de nutrientes, ya que transforman la materia y degradan agentes contaminantes introducidos al suelo (Moreira et al., 2012; Simeto, 2015).

Los hongos son esenciales para la conservación del suelo, así como para las raíces de las plantas, ya que entre $90-95 \%$ de las plantas terrestres crean relaciones simbióticas formando micorrizas para la producción de hormonas, clorofila, tolerancia al estrés abiótico y biótico, y la obtención de nutrientes (en ocasiones provocan un crecimiento más rápido de la raíz para absorber nutrientes como potasio y fósforo), y que el hongo capture el carbono y el nitrógeno necesarios para su desarrollo, favoreciendo la captación de $\mathrm{CO}^{2}$ del planeta (Camargo et al., 2012; Corporación Colombiana de Investigación Agropecuaria, s.f.; Paillacho, 2014).
También pueden ser utilizados en diferentes prácticas culturales, ya que algunos son comestibles, medicinales para combatir enfermedades o utilizarse en la agricultura gracias a la acción de los hongos que combaten otros hongos u patógenos que afectan las plantas, el suelo o insectos (llamados hongos entomopatógenos), así como ser importantes en el arte y el turismo (Gómez et al., 2014).

El objetivo de la investigación es demostrar la importancia de los hongos observados en Finca Boquete-Sibü en los sistemas de producción agrícola, la conservación del suelo y el bienestar social, promover una agricultura $y$ actividades humanas responsables, y fortalecer su uso y protección en los cultivos y la sociedad.

\section{Materiales y métodos}

\section{Sitio de muestreo}

Finca Boquete-Sibü, en El Jardín, es un bosque frondoso y de condiciones topográficas irregulares, ubicado $28 \mathrm{~km}$ norte del Parque Central de Pérez Zeledón en las coordenadas $9^{\circ} 29^{\prime} 41,9^{\prime \prime} \mathrm{N}$ y $83^{\circ} 41^{\prime} 41,1^{\prime \prime}$ W (figura 1), entre los 1500-2 400 m.s.n.m. Se encuentra en la zona de vida bosque pluvial montano bajo, con un tipo de vegetación premontano húmedo y muy húmedo, rodeado de una plantación de mora. El clima cuenta con dos estaciones, la lluviosa (mediados de abril a mediados de diciembre) con altas precipitaciones, y la seca (mediados de diciembre a mediados de abril). La temperatura promedio anual es de $13,7^{\circ}$, con una precipitación de $2455 \mathrm{~mm}$. La mayor precipitación ocurre en octubre, con 385mm (Gamboa, 2013; Climate Data, 2021). 
Figura 1

Sendero de Finca Boquete-Sibü

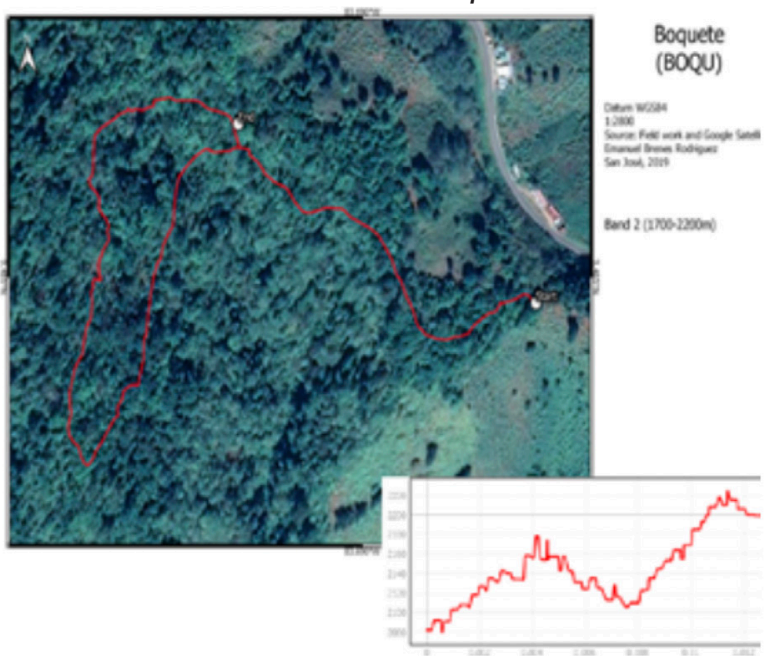

Nota. Fotografía de Emanuel Brenes. Pérez Zeledón, 2019.

\section{Colecta de datos}

Se realizó una visita mensual de campo desde marzo a diciembre de 2020 en Finca Boquete-Sibú, para documentar los cuerpos fructíferos de hongos presentes en un sendero de 1,5 km. Los individuos encontrados fueron ubicados por medio de un waypoint con un GPS "Garmin Etrex 10". Se anotó el género o especie, el sustrato, la altitud, el hábito, los colores, así como otras observaciones ecológicas yde sustrato para su identificación (Vasco et al., 2008).

\section{Resultados y discusión}

\section{Datos obtenidos}

Por medio de las visitas al campo, se pudieron observar diferentes especies de macrohongos. En la tabla 1 se muestran los más abundantes observados en el sendero, un total de 25 individuos de 17 especies diferentes a lo largo de nueve meses.
En la figura 2, se muestran los puntos GPS y el track del sendero donde se observaron los hongos. Al marcar su punto, se anota su identificación, altitud, sustrato, entre otros, además de tomar las fotografías correspondientes.

Figura 2

Ubicación de los hongos observados en el recorrido según su punto GPS

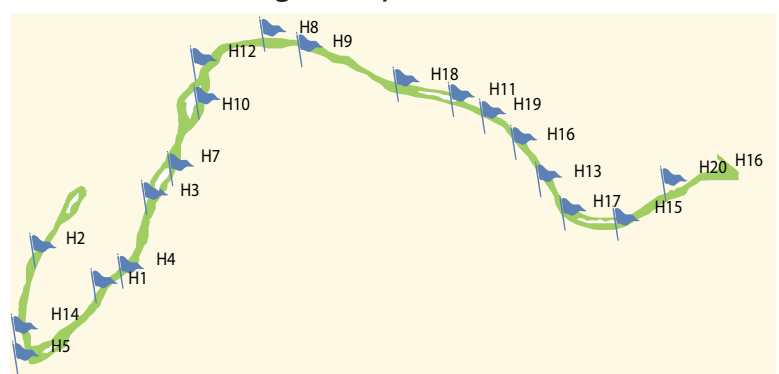

Nota. Finca Boquete-Sibü, Pérez Zeledón, 2020. Elaboración propia.

\section{Degradación del suelo}

Existe gran diversidad de hongos con diferentes funciones y relaciones con su entorno, los cuales permiten el funcionamiento de los ecosistemas y un suelo saludable. La micosfera es el área del sustrato donde se desarrolla el micelio, donde crece el hongo (conformado por filamentos principalmente tubulares llamados hifas, unidades vegetativas del hongo). Es el sitio donde se exponen las secreciones que son producidos por las hifas y se presenta un intercambio entre organismos, que beneficia al suelo y a las plantas (Kuhar et al., 2014).

Aun así, el sustrato se ha visto afectado por prácticas no sostenibles e intensivas, como el pastoreo, la labranza, el uso de agroquímicos, la contaminación, la deforestación, el crecimiento poblacional y el poco conocimiento sobre sus características, lo cual 
Tabla 1

Hongos observados en las visitas de campo en Finca Boquete-Sibü, Pérez Zeledón, 2020

\begin{tabular}{|c|c|c|c|c|}
\hline Fecha & Punto GPS & Nombre Cientifico & Sustrato & $\begin{array}{c}\text { Altitud } \\
\text { (m.s.n.m.) }\end{array}$ \\
\hline $15 / 03 / 2020$ & $\mathrm{H} 1$ & Chlorociboria aeruginascens & Tronco en descomposición (Quercus sp) & 2080 \\
\hline $15 / 03 / 2020$ & $\mathrm{H} 1$ & Russulasp & Suelo & 2080 \\
\hline 05/04/2020 & $\mathrm{H} 2$ & Lentinus sp & Tronco en descomposición & 2124 \\
\hline 05/04/2020 & $\mathrm{H} 2$ & Pleurotus sp & Tronco en descomposición & 2124 \\
\hline 07/05/2020 & $\mathrm{H} 3$ & Ganodermasp & Tronco en descomposición (Quercus sp) & 2092 \\
\hline 07/05/2020 & $\mathrm{H} 4$ & Laetiphorus sulphureus & Tronco en descomposición & 2114 \\
\hline 06/06/2020 & $\mathrm{H} 5$ & Trametes sp & Tronco en descomposición & 2140 \\
\hline 06/06/2020 & $\mathrm{H} 5$ & Hericium sp & Tronco en descomposición & 2140 \\
\hline $06 / 06 / 2020$ & H6 & Hygrocybesp & Suelo & 2115 \\
\hline 06/06/2020 & $\mathrm{H} 7$ & Auricularia delicata & Tronco en descomposición & 2191 \\
\hline $06 / 06 / 2020$ & $\mathrm{H} 8$ & Ganodermasp & Tronco en descomposición & 2133 \\
\hline 06/07/2020 & H9 & Lactifluus indigo & Raíces de Quercus sp & 2190 \\
\hline 06/07/2020 & $\mathrm{H} 10$ & Ramaria cyanocephala & Raíces de árboles & 2120 \\
\hline 06/07/2020 & $\mathrm{H} 11$ & Ramariasp & Tronco en descomposición & 2136 \\
\hline 06/07/2020 & H11 & Boletus sp & Tronco en descomposición & 2136 \\
\hline 06/07/2020 & H12 & Trametes sp & Tronco en descomposición & 2109 \\
\hline 08/08/2020 & $\mathrm{H} 13$ & Russula sp & Suelo y hojarasca & 2141 \\
\hline 08/08/2020 & H14 & Lycoperdon sp & Suelo y hojarasca & 2204 \\
\hline 08/08/2020 & $\mathrm{H} 15$ & Lactifluus indigo & Suelo & 2195 \\
\hline $17 / 09 / 2020$ & H16 & Auricularia delicata & Tronco en descomposición & 2173 \\
\hline 06/10/2020 & $\mathrm{H} 17$ & Ligiella rodrigueziana & Suelo y hojarasca & 2129 \\
\hline $25 / 11 / 2020$ & H18 & Laetiphorus sulphureus & Tronco de descomposición & 2166 \\
\hline $25 / 11 / 2020$ & $\mathrm{H} 19$ & Xylaria sp & Tronco en descomposición & 2201 \\
\hline $25 / 11 / 2020$ & $\mathrm{H} 19$ & Ganoderma sp & Tronco en descomposición & 2201 \\
\hline 07/12/2020 & $\mathrm{H} 2 \mathrm{O}$ & Hygrocybesp & Suelo & 2153 \\
\hline
\end{tabular}

Nota. Elaborada por Krystal Zúñiga Castro con base en la observación de los hongos en Finca Boquete-Sibü, Pérez Zeledón.

afecta la diversidad, provoca la pérdida de fertilidad, la erosión, la alcalinización que daña la producción de alimentos a nivel global y la seguridad alimentaria. Por ello, reducir la labranza en las prácticas agrícolas podría colaborar a la estabilidad de las condiciones del suelo y los ecosistemas, ya que protege las redes fúngicas presentes (Rojas \& Ibarra, 2003, FAO, 2015).

\section{Funciones de los hongos}

Micorrizas: Estas asociaciones son una simbiosis entre hongo y planta,llevan a cabo acciones importantes en el desarrollo natural de los ecosistemas y la agricultura, ya que una de sus funciones es poder colonizar las cortezas y células de las raíces de distintas plantas, aumentando su volumen, 
y permitiéndole adquirir nutrientes minerales, tolerar cambios en el suelo, y una mayor absorción de agua a cambio de compuestos carbonatados que el huésped sintetiza en los procesos de fotosíntesis. A su vez, el hongo obtiene nutrientes orgánicos y un nicho protector (Yoda, 2009; Berdugo, 2009).

La interacción entre plantas y hongos es esencial, ya que la mayoría de las plantas vasculares se vinculan con estos organismos, característica altamente apreciable en zonas cercanas a la cordillera de Talamanca de Costa Rica; en especial, bosques donde predomina Alnus acuminata (jaúl) y se encuentran plantas de la familia Ericaceae, ya que se pueden apreciar gran cantidad de hongos alrededor de estos sustratos (Vargas, 2015).
Un árbol característico del sitio estudiado, e importante ecológicamente, es el roble (Quercus $s p$ ) de la familia Fagaceae, son de gran tamaño, y poseen una corteza fuerte con condiciones óptimas para interacciones simbióticas con algunos hongos, así como ser hospedero de especies como bromelias, u orquídeas, las cuales forman interacciones con hongos en su desarrollo inicial de crecimiento para obtener nutrientes y carbono, estas micorrizas son llamadas endomicorrizas orquideoides (Marañón, 2011, Andrade, 2010). Este árbol presenta interacciones con algunos hongos encontrados, como Boletus sp, Russula sp (figura 3 a.), Ramaria cyanocephala "hongos Coral" (figura 3c.), Lactifluus indigo (figura 7 a.), colaborador de la restauración del ecosistema, y Auricularia delicata (figura 7

Figura 3

Hongos formadores de micorrizas

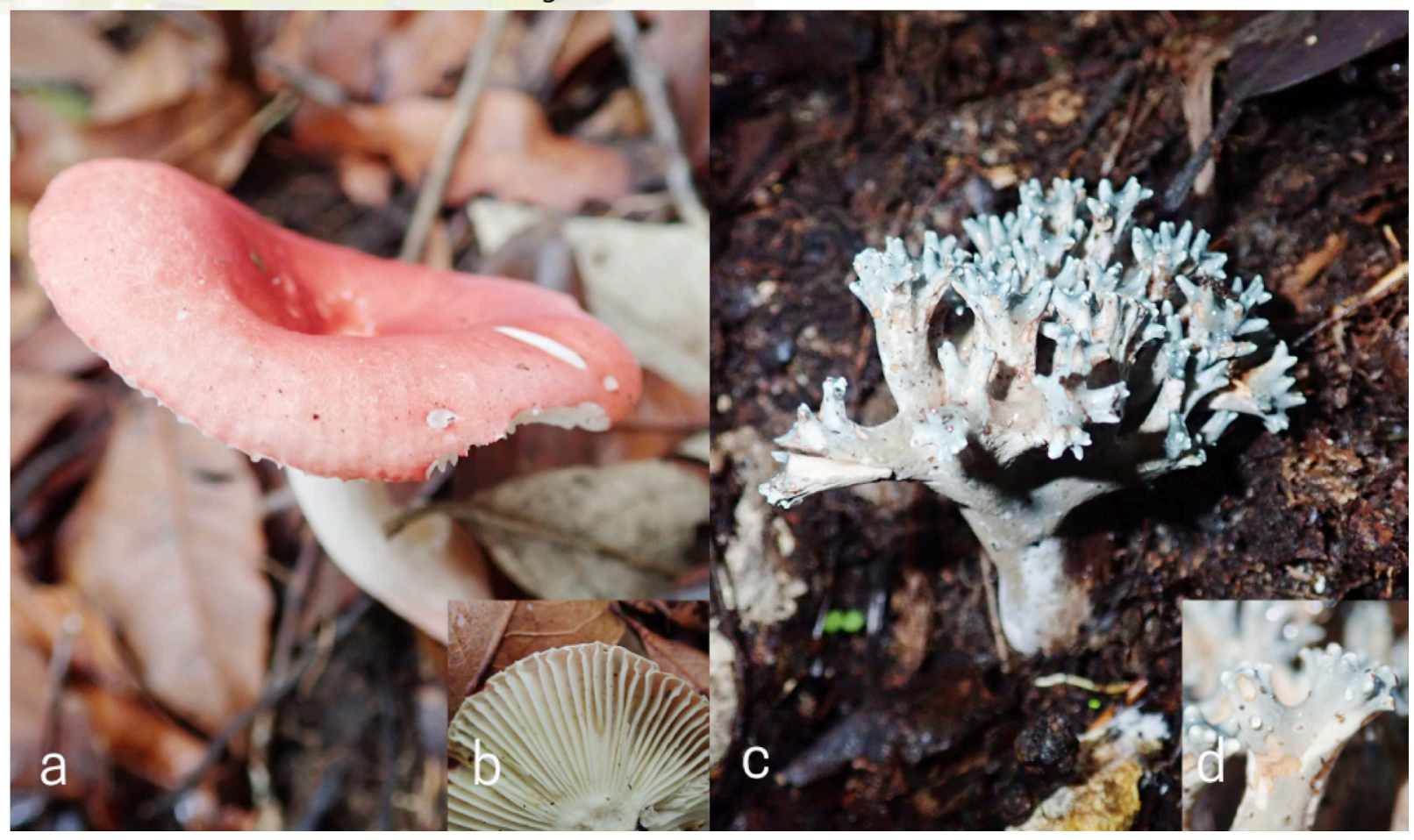

Nota. Selección: $\boldsymbol{a}$-Russula sp., $\boldsymbol{b}$ - Himenóforos de Russula sp, c-Ramaria cyanocephala, $\boldsymbol{d}$-Detalle basidioma en forma ramarioide de R. cyanocephala. Fotografías de Krystal Zúñiga Castro. Finca Boquete-Sibü, Pérez Zeledón, 2020. 
c.) hongo solubilizador de los nutrientes de la planta (Velasco, 2019; Smith \& Smith, 2007).

Algunas micorrizas forman una red por medio del micelio, la cual permite conectar a los árboles a través del bosque, compartir nutrientes, advertir sobre ataques de plagas y protegerse. Otras otorgan mayor resistencia a la planta y al suelo ante el estrés hídrico, los patógenos y brindan ventajas en la absorción y transporte de iones y elementos esenciales para su desarrollo, en especial del fósforo y el nitrógeno, así como de potasio, zinc y cobre, ya que generan transportadores de alta afinidad hacia estos elementos, brindando energía adicional hacia la planta. A su vez, reducen la captación de otros elementos que pueden ser tóxicos, como el manganeso (Azcón \& Talón, 2008; Borie et al., 2000).

Fijadores de nitrógeno: El nitrógeno en la agricultura es uno de los principales componentes, ya que su deficiencia provoca clorosis (coloración amarilla, verde pálido o blanquecina en el follaje y baja producción o destrucción de clorofila de la hoja, principalmente por la falta de hierro), debilitación y falta de desarrollo, pues la mayoría de las plantas no pueden metabolizar el nitrógeno por sí solos. Algunas bacterias tienen la capacidad de capturar el nitrógeno atmosférico y disponerlo tanto para microorganismos del suelo como para las plantas, por ende, algunas especies de hongos forman relaciones simbióticas con estas bacterias y a su vez con las plantas y facilitan además el transporte de nitrógeno, fósforo y agua. Varios autores han demostrado que en sustratos donde se desarrollan los hongos se encuentran valores de nitrógeno que oscilan entre $0,1 \%$ y $1 \%$,se considera que estos podrían ser capaces de fijar nitrógeno atmosférico (Sánchez, 2017).
Protección ante hongos fitopatógenos y estimuladores en el crecimiento de plantas: LoS hongos del género Trichoderma pueden encontrarse principalmente en el suelo, son de crecimiento rápido, poseen enzimas promotoras de crecimiento vegetal por medio de la producción de auxinas, giberelinas y ácidos orgánicos. Su aislamiento e inoculación para utilizarse en la agricultura pueden disminuir el pH del suelo, mejorar la absorción de fósforo, la capacidad fotosintética, el crecimiento; incrementar la tolerancia a los cambios del suelo, controlar patógenos en los cultivos, entre otros (Hernández et al., 2019).

Los hongos citados, así como del género Gliocladium, se utilizan como biocontroladores contra hongos fitopatógenos, los cuales provocan enfermedades totales o parciales en las estructuras de las plantas, alteran su desarrollo, producen necrosis del tejido, desfiguraciones en los frutos y productos subterráneos, podredumbre, y al ser consumidos pueden provocar riesgos en la salud; además de poder ser capaces de producir micotoxinas que se distribuyen con facilidad en el sustrato (Carreras et al., 2013; Trigos et al., 2018).

El Gliocladium roseum es utilizado como biocontrolador contra Botrytis cinerea, hongo parasitario que afecta a frutos y flores de las plantas. Al utilizarlo se evita el uso de fungicidas, y su eficacia es igual o más efectiva que los tratamientos con químicos. Además, compite por el sustrato colonizando más rápido y evita la acción del hongo parásito $B$. cinerea (Chaves, 2004).

Controladores de nematodos y patógenos: El suelo posee diferentes organismos, entre ellos los nematodos, estos poseen un estilete en la boca, el cual utilizan para perforar las células radicales de las plantas. En la mayoría de casos, 
atacan las raíces; sin embargo, también pueden hallarse en partes aéreas de las plantas, lo cual facilita la entrada de agentes patógenos que causan daños en su interior (Piedra, 2008).

Como medida para contrarrestar este tipo de plagas, se puede implementar el uso de hongos hematófagos, encargados de controlar biológicamente los nematodos. Una especie con esta función es Pleurotus sp, uno de los hongos más estudiados por los científicos. Se caracteriza por ser productor de toxinas que inmovilizan a los nematodos antes de infectarlos (López \& Hans, 2001). Una vez el nematodo se pone en contacto con la toxina, se inmoviliza y las hifas del hongo crecen por medio de la boca del nematodo (Piedra, 2015).

Algunos hongos hematófagos, que habitan suelos con alto contenido de materia orgánica, se utilizan para combatir diferentes tipos de nematodos fitoparásitos, tal es el caso como algunos hongos del género Trichoderma, que al usarse con metabolitos de Laetiporus sulphureus (figura 8) permiten combatir nematodos fitoparásitos comunes en el cultivo de lechuga, como es el caso de Meloidogyne sp. y Helicotylenchus sp (Piedra \& Vargas, 2016).

El hongo Gliocladium sp es de los más comunes utilizados en el biocontrol de patógenos vegetales y comparte nichos ecológicos similares al de Trichoderma sp. A partir de Gliocladium se han formulado productos que protegen la raíz de las plantas, y se logra disminuir el uso de aplicaciones químicas (Cano, 2011; Van, 2014).

Biorremediadores del suelo: Los hongos biorremediadores permiten la degradación de agentes contaminantes en el suelo. Un ejemploson los delosgéneros Ganoderma (figura4) y Pleurotus, los cuales poseen enzimas de lacasa y actúan en la degradación de residuos de lignina (biopolímero que refuerza la pared celular y brinda resistencia a las plantas), permitiendo los procesos para la biorremediación enzimática de los suelos (Valdés, 2018). El Pleurotus es el precursor principal para la reserva de materia orgánica en el suelo, puede mineralizar agentes contaminantes del suelo al introducir sus enzimas, y regula el ciclo de carbono, siendo un requisito para la hidrólisis de otros componentes de la biomasa vegetal, su estructura dependerá de la especie de planta, tasa fotosintética y estado fenológico (Coello, 2011; Ortiz, 2009; Cerón \& Melgarejo, 2005).

Hongos como los Trametes son importantes en la eliminación de desechos y reciclaje de materia, pues su nutrición ocurre por medio de la combustión de la madera, siendo degradadores de contaminantes ambientales como los plaguicidas o explosivos, y poseen un uso potencial en la biorremediación, al igual que el Trichoderma, ya que crece en varios sustratos y degrada hidrocarburos, polisacáridos, entre otros (Estrada et al., 2016, Cano 2011).

\section{Figura 4}

Hongos para biorremedación de suelos

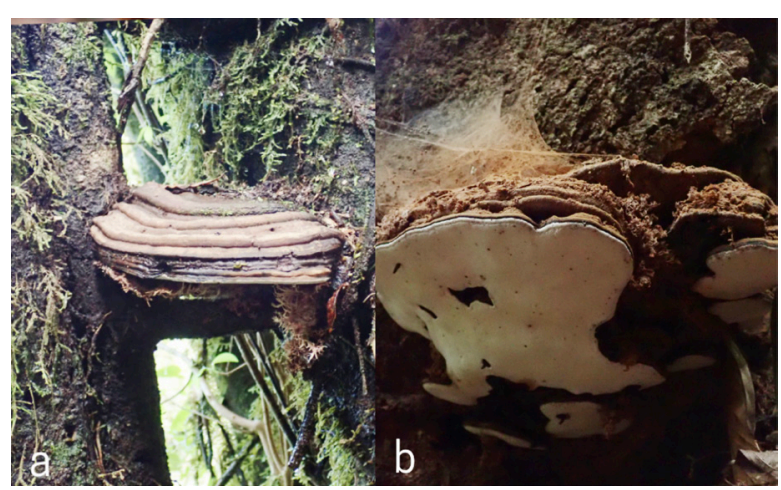

Nota. Selección: $\boldsymbol{a}$-Ganoderma sp., $\boldsymbol{b}$-Detalles de Ganoderma $s p$. Fotografía por Krystal Zúñiga Castro. Finca Boquete-Sibü, Pérez Zeledón, 2020. 


\section{Figura 5}

Hongos descomponedores de materia y reciclaje de nutrientes

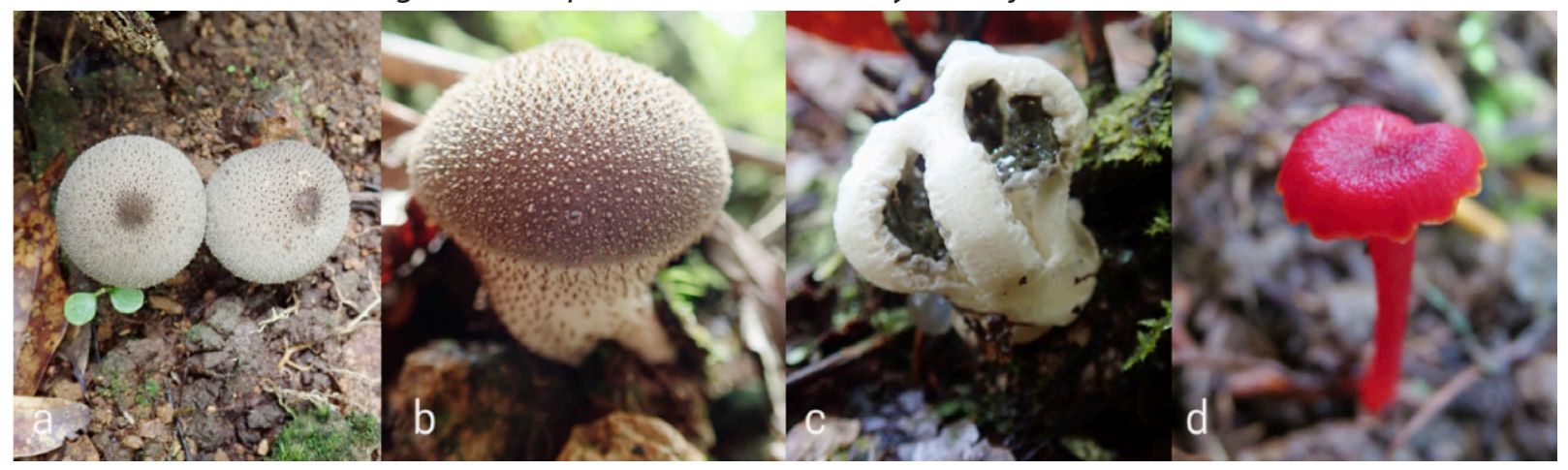

Nota. Selección: $\boldsymbol{a}$-Lycoperdon sp. $\boldsymbol{b}$-Forma gasteroide de Lycoperdon sp. c-Ligiella rodrigueziana. $\boldsymbol{d}$ - Hygrocybe sp. Fotografía de Krystal Zúñiga Castro. Finca Boquete-Sibü, Pérez Zeledón, 2020.

Descomponedores de materia orgánica y reciclaje de nutrientes: Los hongos saprófitos son individuos que obtienen su nutrición por medio de los residuos de la materia orgánica, colaborando con la descomposición. Hongos como Thichoderma y Lycoperdon son descomponedores de materia leñosa y herbácea. Lycoperdon (figura 5 a.), posee una morfología gasteroide (forma de estómago), y una de sus principales funciones es degradar la materia orgánica y el reciclaje de nutrientes, provocando que el suelo sea más productivo (Salinas \& Gómez, s.f.). Especies como Ligiella rodrigueziana (figura 5 c.) son indicadores de bosques ricos en materia orgánica para su descomposición, como Hericium y Auricularia, ya que contribuyen con el reciclaje de nutrientes (Sáenz \& Nassar, 1981)

El género Hygrocybe (figura 5 d.) se presenta en suelos con gran cantidad de materia vegetal, atacan los restos de las hojas con una red de hifas, lo cual provoca la mineralización foliar. Son indicadores de la calidad del suelo, ya que se encuentran en suelos pobres en nitrógeno y otros nutrientes, donde sus cuerpos fructíferos son los más dominantes. Los hongos que pertenecen a la familia Hygrophoracea, son utilizados en Europa del Norte y Groenlandia como un grupo con propósitos de conservación (Lodge et al., 2013).
El Ganoderma, el Pleurotus y el Lentinus (figura 6 a.) son utilizados frecuentemente para el reciclaje de desechos lignocelulósicos y la transformación de contaminantes como hidrocarburos, fertilizantes o productos farmacéuticos, ya que además poseen un crecimiento rápido del micelio, reducen las bacterias y tienen la capacidad de crecer en diferentes sustratos y condiciones ambientales (Sánchez \& Royse, 2017; Gaia et al., 2015).

En el caso de Xylaria $s p$ "dedos de muerto" (Figura 6 b.), o Trametes $s p$, son descomponedores importantes por su gran producción de enzimas como lacasa, degradan el material orgánico, por lo que han sido utilizados para aplicaciones biotecnológicas en la agricultura (Sandoval et al., 2017; Estrada et al., 2016).

El Ganoderma también es de los descomponedores más abundantes, crecen en ambientes tropicales y causan una muerte blanca en la raíz y el duramen (parte central que brinda resistencia a las ramas o tallos formando por células muertas y sin actividad conductora), hasta provocar su degradación. Algunas especies de este género poseen propiedades antioxidantes de gran importancia medicinal (Hernández, s.f.; Islas et al., 2017). 
Figura 6

Hongos descomponedores de materia y recicladores de nutrientes

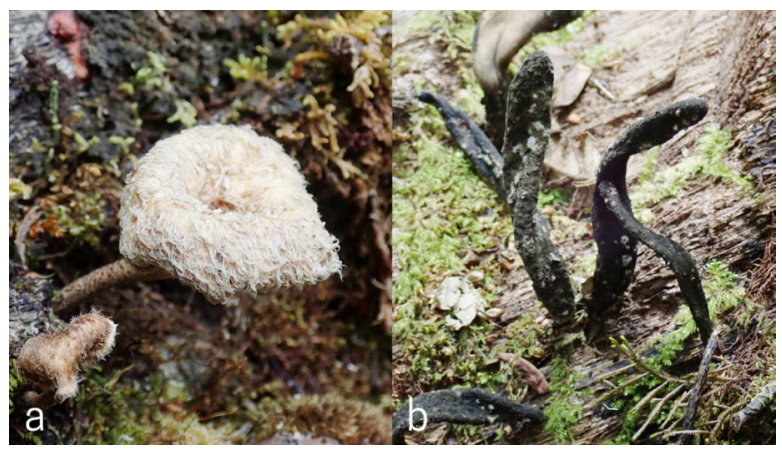

Nota. Selección: $\boldsymbol{a}$-Lentinus sp, $\boldsymbol{b}$-Xylaria sp. Fotografía de Krystal Zúñiga Castro. Finca Boquete-Sibü, Pérez Zeledón, 2020.

Hongos comestibles, medicinales, artísticos y turísticos: Los hongos también pueden ser de gran atracción turística gracias al micoturismo, actividad enfocada en su apreciación, la micogastronomía y, en ocasiones, su recolección. Es una práctica cultural, por el interés en conocer los sitios de donde proceden los hongos (principalmente comestible), su preparación y ética de consumo. Para un micoturismo sostenible, se necesita conocimiento previo de los hongos, y un sistema de información micológica por medio de métodos de georreferenciación e indicadores etnobiológicos, que determinarán cuáles hongos pueden ser utilizados y su disponibilidad (Thomé, 2014).

Algunos hongos también pueden ser comestibles o medicinales, como Hericium (Figura 9), uno de los más importantes en la medicina y la gastronomía, el cual presenta gran contenido en fibra, es regenerador de mucosa intestinal, promueve el desarrollo cerebral, es antitumoral y se utiliza en enfermedades neurodegenerativas y del aparato digestivo (Hifas da Terra Mushroom Bioscience, 2020). Algunos como Auricularia delicata (Figura 7 c.), el cual es gelatinoso y presenta forma de oreja, a pesar de ser comestible, por su bajo contenido nutricional al ser 90,2\% agua, no es de gran utilidad en gastronomía (Melgarejo, 2014).

Hongos comestibles como Lactifluus indigo, abundante principalmente en épocas lluviosas, forman micorrizas importantes, y presenta un alto grado nutricional. Estudios recientes muestran que también posee propiedades farmacéuticas, anticancerígenas y microbianas (Cano \& Romero, 2016). Se caracteriza por la presencia de látex en los carpóforos (cuerpo fructífero del hongo constituido por hifas, con colores y formas según su especie), los cuales cambian de color al cortarlos (Ardiles et al., 2009). El látex contiene diferentes sesquiterpenos, entre ellos

Figura 7

Hongos comestibles

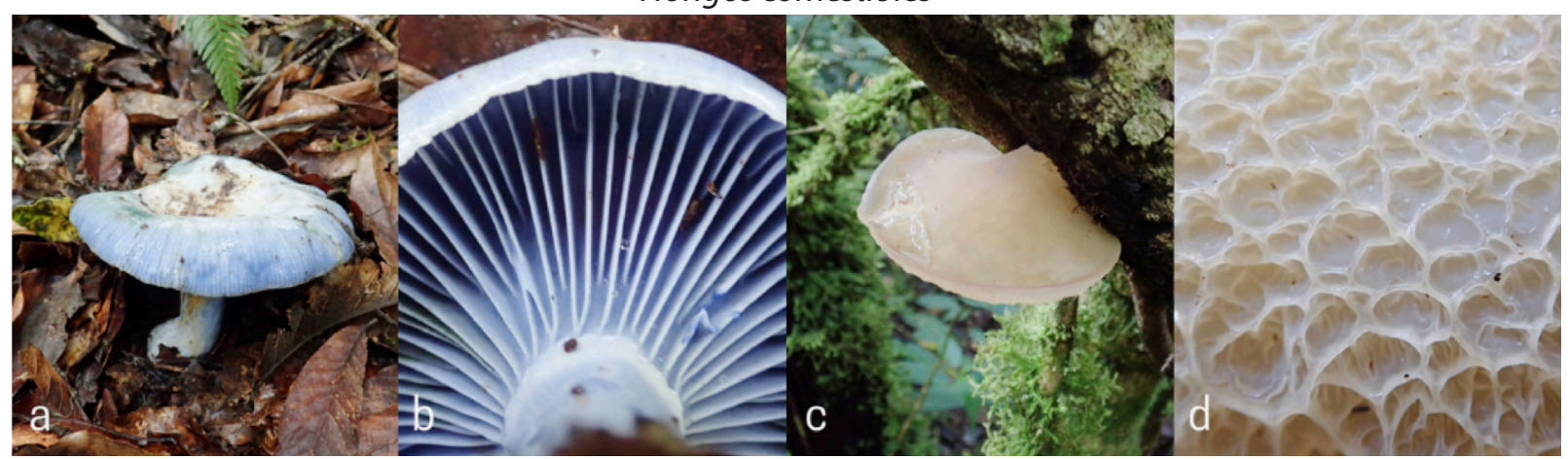

Nota. Selección: $\boldsymbol{a}$-Lactifluus indigo, $\boldsymbol{b}$-Himenóforo de L. indigo, c-Auricularia delicata, $\boldsymbol{d}$-Detalle de A. delicata. Fotografías de Krystal Zúñiga Castro. Finca Boquete-Sibü, Pérez Zeledón, 2020. 
estearoildeterrol, siendo este el principal en los carpórofos, y el responsable de su color azul. Los sesquiterpenos actúan como defensa química contra depredadores ya que presentan gran actividad microbiana, por lo cual su consumo directo, sin una cocción previa, podría provocar una intoxicación (Pomilio, 2019).

Laetiporus sulphureus, llamado popularmente como "pollo del bosque", contiene gran cantidad de proteínas, carbohidratos y un agradable sabor muy característico de su especie, posee propiedades antimicrobianas, antioxidantes y antitumorales. No debe de consumirse en estado inmaduro, y se recomienda no mezclarlo con alcohol (Rodríguez, 2019).

El hongo Chlorociboria aeruginascens (figura 10 a.), que se encuentra principalmente en árboles de la familia Fagaceae (Quercus $s p$ ), ha sido utilizados a través del tiempo para crear obras de arte, por el color que toma el sustrato al descomponerse. Artistas italianos, hace más de 500 años, crearon un método artístico llamado Intarsia, en el cual se utiliza la madera con la presencia del pigmento azul-verde llamado xylindein liberado por el hongo (Robinson et al., 2012).
Figura 8

Hongo comestible

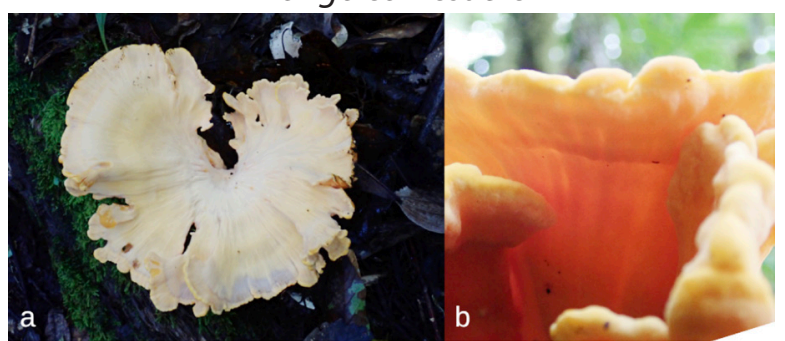

Nota. $\boldsymbol{a}$-Laetiporus sulphureus, $\boldsymbol{b}$-Detalles L. sulphureus. Fotografías de Krystal Zúñiga Castro. Finca Boquete-Sibü, Pérez Zeledón, 2020.

Figura 9

Hongo comestible y medicinal

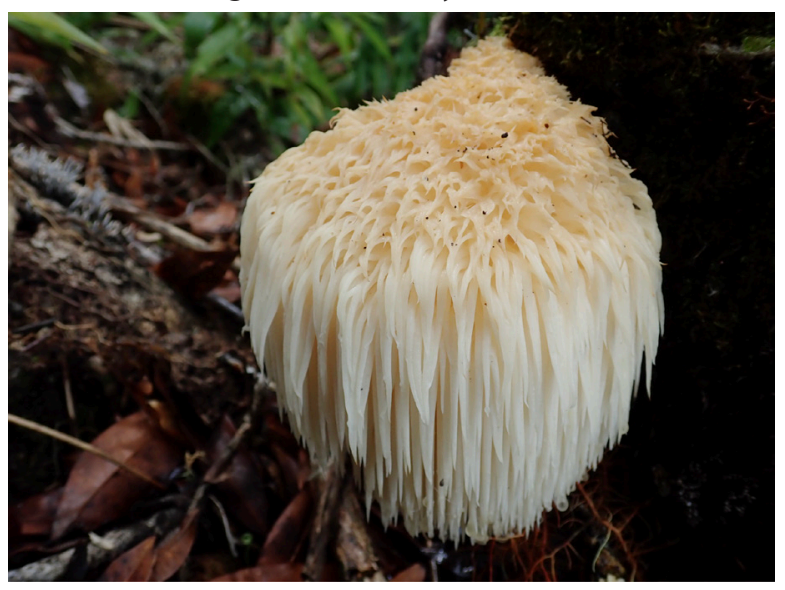

Nota. Hericium sp. Fotografías de Krystal Zúñiga Castro. Finca Boquete-Sibü, Pérez Zeledón, 2020.

Figura 10

Hongos para técnicas artísticas

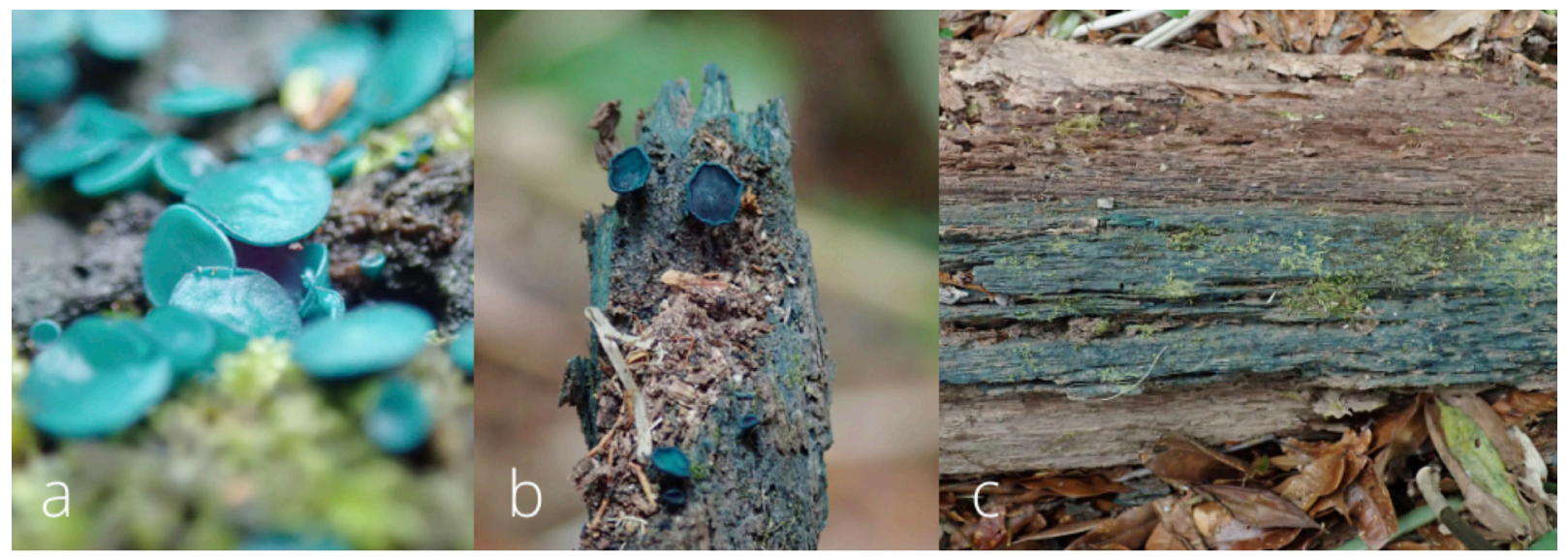

Nota. Selección: $\boldsymbol{a}$-Chlorociboria aeruginascens, $\boldsymbol{b}$ - c. aeruginascens en Quercus sp, c- Pigmento xylindein en Quercus sp producto de la descomposición de C.aeruginascens. Fotografías de Krystal Zúñiga Castro. Finca Boquete, Pérez Zeledón, 2020. 


\section{Conclusiones}

El uso de hongos en la agricultura es uno de los avances de mayor importancia en la búsqueda de soluciones y controladores naturales para mejorar la salud del suelo y fortalecer los cultivos, ya que poseen la capacidad de eliminar patógenos, controlar nematodos y enfermedades, por lo cual se logra reducir la cantidad de agroquímicos y fármacos inhibidores en las plantas.

Además, es una alternativa eficiente y ecológica que brinda numerosos beneficios al suelo y las plantas, tales como mejorar su nivel nutricional, la obtención de nitrógeno, de agua, favorecen el crecimiento, el desarrollo y la comunicación por medio de las redes formadas por el micelio.

Su protección puede impactar a nivel social y ambiental por sus funciones en la descomposición de la materia y la biorremediación de suelos, además de ser utilizados como alimento, medicina, en el arte y el turismo. Fortalecen así la seguridad alimentaria, el desarrollo y la conservación del ambiente.

\section{Referencias}

Andrade, A. (2010). Micorrizas: antigua interacción entre planta y hongos. Ciencia, vol. 61 (4), 84-90. https://www.revistaciencia.amc.edu.mx/images/ revista/61 4/PDF/11 MICORRIZAS.pdf

Ardiles, V., Osorio, F., Barrera, E. (2009). Gayana Botánica, 66 (1), 84-91. https://scielo.conicyt.cl/scielo.php?script=sci arttext\&pi$d=\$ 0717-66432009000100008$

Azcón, J., Talón, M. (2008), Fundamentos de fisiología vegetal. Madrid, España. McGraw-Hill. http://exa.unne.edu.ar/biologia/fisiologia.vegetal/ FundamentosdeFisiologiaVegetal2008Azcon.pdf
Berdugo, S. E. (2009). El uso de hongos micorrízicos arbusculares como una alternativa para la agricultura. Biotecnología en el Sector Agropecuario y Agroindustrial, 7(1), 123-132. https://revistas.unicauca.edu.co/index.php/biotecnologia/article/ $\underline{\text { view/706 }}$

Borie, F.; Morales, R.; Castillo, C. (2000). Relación entre densidad de hifas de hongos micorrizógenos arbusculares y producción de glomalina con las características físicas y químicas de suelos bajo cero labranza. Revista Chilena de Historia Natural, 73 (4), 749-756. https://scielo.conicyt.cl/scielo.php?scrip$\underline{\mathrm{t}=\text { sci } \text { arttext\&pid }=\text { S0716-078X2000000400017 }}$

Burbano, H., (2016). El suelo y su relación con los servicios ecosistémicos y la seguridad alimentaria. Revista de Ciencias Agrícolas, 33 (2), 117-124. http://www. scielo.org.co/pdf/rcia/v33n2/v33n2a11.pdf

Camargo, S.; Montaño, M.; De la Rosa, C; Montaño, S. (2012). Micorrizas: una gran unión debajo del suelo. Revista Digital Universitaria, vol 13 (7), 4. http://www.revista.unam.mx/vol.13/num7/art72/

Cano, A., Romero, L. (2016). Valor económico, nutricional y medicinal de hongos comestibles silvestres. Revista Chilena de Nutrición, 43, (1), 75-80. https://www.redalyc.org/pdf/469/46946023011.pdf

Cano, M. (2011). Interacción de microorganismos benéficos en plantas: micorrizas, Trichoderma spp. y Pseudomonas spp. Una revisión. Revista UDCA Actualidad y Divulgación Científica, 14, (2), 15 31. http://www.scielo.org.co/pdf/rudca/v14n2/ $\underline{v 14 n 2 a 03 . p d f}$

Carreras, N., Hernández, E., Sánchez, D. (2013). Conociendo a los hongos fitopatógenos. Ciencia Hoy. https://www.inecol.mx/inecol/index.php/es/201306-05-10-34-10/17-ciencia-hoy/1042-conociendo-alos-hongos-fitopatogenos

Cerón, L.; Melgarejo, L. (2005), Enzimas Del Suelo: Indicadores de salud y calidad, Acta Biológica Colombiana, 10 (1): 5-18. https://www.redalyc.org/ pdf/3190/319028576001.pdf

Chaves, N., Wang, A.(2004). Combate del moho gris (Botrytis cinerea) de la fresa Gliocladium roseum. Agronomía Costarricense. 28(2): 73-85. https://www.mag.go.cr/ rev agr/v28n02 073.pdf 
Climate Data (2021). Jardín Clima, Costa Rica. ClimateData.org. Consultado el 29 de enero de 2021. https://es.climate-data.org/america-del-norte/ costa-rica/san-jose/jardin-719097/\#weather

Coello, J. (2011). Aplicación del Hongo Pleurotus ostreatus como alternativa para la biorremediación de suelos contaminados con metales pesados. Tesis de Licenciatura. Guayaquil, Ecuador, Escuela Superior Politécnica del Litoral, Guayaquil. Ecuador. https://www.dspace.espol.edu.ec/bitstream/123456789/21150/1/D-92862.pdf

Corporación Colombiana de Investigación Agropecuaria. (s.f.). Las Micorrizas: una opción sostenible de manejo de suelos y nutrición de las plantas. Bogotá, Colombia: Corpoica. 2 p. https://books. google.co.cr/books?id=bTmd - XYrPwC\&pg=

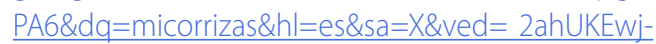
68LL68p3tAhXFQjABHfIKBfIQ6AEwAHoECAUQAg\#$\underline{v=o n e p a g e \& q=\text { micorrizas } \& f=\text { false }}$

Gamboa, F. (2013). Promoción de herramientas organizativas, de planificación y fomento del capital social para el desarrollo comunal: el caso de la comunidad de El Jardín, Paramo, Pérez Zeledón (Marzo-Diciembre, 2013). Trabajo final de graduación. Universidad de Costa Rica. http://repositorio.sibdi.ucr.ac.cr:8080/ispui/bitstream/123456789/2553/1/38701.pdf

Gómez, H., Zapata, A., Torres, E., Tenorio, M., (2014). Manual de producción y uso de hongos entomopatógenos. Servicio Nacional de Sanidad Agraria de Perú. https://www.senasa.gob.pe/senasa/descargasarchivos/2017/09/Manual-de-Producci\%C3\%B3n-y-Usode-Hongos-Entomopat\%C3\%B3genos.pdf

Hernández, A. (s.f.). Albura y Duramen. Ciencias en pocas palabras, 232 (26), 415. https://www.sabermas.umich. mx/archivo/la-ciencia-en-pocas-palabras/232-numero-26/415-albura-y-duramen.html

Hernández, D., Ferrera, R., Alarcón, A. (2019). Trichoderma: Importancia Agrícola, biotecnológica, y sistemas de fermentación para producir biomasa y enzimas de interés industrial. Chil.j. agric. anim. Sci, 35, (1). 98-112. https://scielo.conicyt.cl/scielo.php?script=sci arttext\&pid=S0719-38902019000100098\#B67

Hifas da Terra Mushroom Bioscience (2020). Melena de León. Hifas da Terra Mushroom Bioscience. https://hifasdaterra.com/blog/hongos/ melena-de-leon-hericium-erinaceus/
Islas, M., Castañeda, A., Álvarez, A., Valenzuela, R., Romero, L., Torres, J. (2017). Estudio preliminar de la actividad antioxidante de tres especies del género Ganoderma (Polyporaceae) nativas del estado de Hidalgo, México. Revista Mexicana de Micología, Xalapa, 46, 37-45. http://www. scielo.org. $\mathrm{mx} /$ scielo.php?script=sci arttext\&pi$\underline{d=S 0187-31802017000200037 \& \operatorname{lng}=e s \& n r m=i s 0}$

Kuhar, F.; Castiglia, V.; Papinutti, L. (2013). Reino Fungi: Morfología y estructuras de los hongos. Revista Boletín Biológica, 48 (7), 11-18. https://core.ac.uk/ download/pdf/52479411.pdf

Lodge, D., Padamsee, M., Matheny, P., Aime, M., Cantrell, S., Boertmann, D., Kovalenko, A., Vizzini, A., Dentinger, B., Kirk, P., Ainsworth, M., Moncalvo, J., Vilgalys, R., Larsson, E., Lücking, R,Griffith, G., Smith, M., Norvell, L., Desjardin, D., Redhead, Scott., Ovrebo, C., Lickey, E., Ercole, E. Hughes, K., Courtecuisse, R., Young, A., Binder, M., Minnis, A. Lindner, D., Ortiz, S., Haight, J.,Læssøe, T., Baroni, T., Geml, J., Hattori, T. (2014). Molecular phylogeny, morphology, pigment chemistry and ecology in Hygrophoraceae (Agaricales). Fungal Diversity, 64, (1). 1-99. https://link.springer.com/article/10.1007/ $\underline{\text { s13225-013-0259-0 }}$

Melgarejo, E. (2014). Dos hongos silvestres comestibles de la localidad de Incachaca, Cochabamba (Yungas de Bolivia). Acta Nova, 6, (4), 384-394. http://www. scielo.org.bo/pdf/ran/v6n4/v6n4 a04.pdf

Moreira, F., Huising, E., Bignell, D. (2012). Manual de biología de suelos tropicales. Instituto Nacional de Ecología, México. México. https://books.google. co.cr/books?id=m-QMZaBiPOYC\&printsec=frontcover\&hl=es\&source=gbs_ge_summary_r\&cad=0\#$\mathrm{v}=$ onepage $\& \mathrm{q} \& \mathrm{f}=\mathrm{false}$

Organización de las Naciones Unidas para la Alimentación y la Agricultura (4 de diciembre 2015). Los suelos están en peligro, pero la degradación puede revertirse. http://www.fao.org/news/story/es/item/357165/ icode/

Ortiz, M. (2009). Aproximaciones a la comprensión de la degradación de la lignina. Orinoquia, 13 (2), 137-144. https://www.redalyc.org/pdf/896/89613728007.pdf 
Paillacho, F. (2014). Evaluación de la efectividad de las micorrizas arbusculares nativas sobre el desarrollo y estado nutritivo del palmito (Bactris gasipaes hbk) en etapa de vivero, en santo domingo de los tsáchilas. Tesis de maestría. Escuela Politécnica del Ejército, Ecuador. http://repositorio.espe.edu.ec/xmlui/ bitstream/handle/21000/2892/T-ESPE-IASA\%20II002332.pdf? sequence $=1 \&$ isAllowed $=y$

Piedra, R., Vargas, C. (2016). Hongos hematófagos en el combate de nematodos fitoparásitos asociados al cultivo de lechuga. Alcances Tecnológicos, 11(1): 41- 48. http://revista.inta.go.cr/index.php/ alcances tecnologicos/article/download/26/14/

Piedra, R. (2008). Manejo biológico de nematodos fitoparásitos con hongos y bacterias, Tecnología en Marcha, 21(1): 123 - 132. https://revistas.tec.ac.cr/index.php/tec marcha/article/view/1345

Piedra, R. (2015). Guía de muestreo de nematodos fitoparásitos en cultivos agrícolas, Instituto Nacional de Innovación y Transferencia en Tecnología Agropecuaria San José, C.R., 22 p. http://www.mag. go.cr/bibliotecavirtual/H10-10789.PDF

Pomilio, A., Maris, S., Alonso, A. (2019). Micetismos. Parte 3. Síndromes tempranos gastrointestinales. Toxicología, 53, (2), 14-19. https://www.redalyc.org/ jatsRepo/535/53560335009/html/index.html

Robinson, S., Tudor, D., Snider, H., Cooper, P. (2012). Stimulating growth and xylindein production of Chlorociboria aeruginascens in agar-based systems. AMB Express Journal, 2 (15), 1-7. https://www. ncbi.n/m.nih.gov/pmc/articles/PMC3350399/

Rodríguez, I. (2019). El pollo del bosque no es un animal. Revista de Biología Tropical (en línea). https://revistas.ucr.ac.cr/index.php/rbt/article/view/39416

Sáenz, J., Nassar, M. (1981). Hongos de Costa Rica: Familias Phallaceae y Clathraceae. Revista de Biología Tropical. 30, (1), 41-52. https://tropicalstudies.org/rbt/attachments/volumes/vol30-1/05-Saenz-Hongos.pdf

Salinas, M., Gómez, R. (s.f.). Los estómagos de los bosques, hongos gasteroides. Saber Más, Revista de Divulgación de la Universidad Michoacana de San Nicolás de Hidalgo. 299, (52). 542. https://www. sabermas.umich.mx/archivo/articulos/299-numero-35/542-los-estomagos-del-bosque-hongos-gasteroides.html

58
Sánchez, J., Royse, D. (2017). La biología, el cultivo y las propiedades nutricionales y medicinales de las setas Pleurotus sp. El Colegio de la Frontera Sur, San Cristóbal de las Casas, Chiapas, México. http://aleph.ecosur.mx:8991/exlibris/aleph/a22 1/apache media/ TVYDRJDU7JPAQEH4LF688NSKFF8S7Q.pdf

Sandoval, J.; Ochoa, F;; Torres, E. (2017). Evaluación de diferentes métodos de extracción de ARN a partir del hongo nativo Xylaria sp. Revista Colombiana de Biotecnología, 19 (1), 42-54. https://www.researchgate.net/publication/318006608 Evaluacion de diferentes metodos de extraccion de ARN a partir del hongo nativo Xylaria sp

Smith, T.; Smith, R. (2007). Ecología. Madrid, España: Pearson Educación.

Trigos, A.; Ramírez, K.; Salinas, A. (2018).Presencia de hongos fitopatógenos en frutas y hortalizas y su relación en la seguridad alimentaria. Revista Mexicana de Micología, 28, 125-129. http://www. scielo.org. mx/scielo.php? script=sci arttext\&pi$\underline{d=S 0187-31802008000300015}$

Thomé, H. (2014). Turismo micológico, una nueva mirada al bosque. Ciencia y Desarrollo, 25, 10-17. https://www.cyd.conacyt.gob.mx/?p=articulo\&id=25

Valdés, S. (2018). Aislamiento y purificación parcial de enzimas microbianas de tipo Lacasa, para la degradación de desechos de piña, banano y caña, Universidad de Costa Rica. Tesis Lic. Heredia, CR. UNA. https://repositorio.una.ac.cr/bitstream/handle/11056/14365/Tesis\%20Sandra\%20Valdés\%20Diaz. pdf? sequence $=1$

Van, A. (2014). Encyclopedia of Agriculture and Food Systems. ScienceDirect. https://www.sciencedirect.com/ topics/agricultural-and-biological-sciences/ gliocladium

Vargas, G. (2015). Botánica general: Desde los musgos hasta los árboles. San José, CR: EUNED.

Vasco, A.; Suaza, S.; Castaño, M.; Franco, A. (2008). Conocimiento etnoecológico de los hongos entre los indígenas Uitoto, Muinane y Andoke de la Amazonia de Colombia.

Velasco, M. (2019). Hongos en rodales de pinos-encino en la comunidad de Miguel Hidalgo. Municipio de San Antonio Huitepec, Distrito de Zaachila, Oaxaca. Tesis de Maestría. Universidad Autónoma de Chepingo, Oaxaca, México. http://dicifo.chapingo. $\underline{\mathrm{mx} / \mathrm{pdf} / \mathrm{tesis}}$ 